\title{
Knockout of SIRT4 decreases chemosensitivity to 5-FU in colorectal cancer cells
}

\author{
YUANYUAN ZHU ${ }^{1}$, GUANGYU WANG ${ }^{1}$, XIAOBO LI ${ }^{2}$, TIANZHEN WANG ${ }^{2}$, \\ MINGJIAO WENG $^{2}$ and YANQIAO ZHANG $^{1}$ \\ Departments of ${ }^{1}$ Gastrointestinal Medical Oncology and ${ }^{2}$ Pathology, \\ Harbin Medical University Cancer Hospital, Harbin, Heilongjiang 150081, P.R. China
}

Received July 24, 2017; Accepted April 16, 2018

DOI: $10.3892 / \mathrm{ol} .2018 .8850$

\begin{abstract}
Previous studies demonstrated that sirtuin (SIRT) 4 is aberrantly expressed in human malignant tumors and is associated with poor prognosis in patients with colorectal cancer. However, the role of SIRT4 in the progression of human colorectal cancer (CRC) and in chemotherapy remains unclear. In the present study, the expression of SIRT4 in CRC tissues and the effect of SIRT4 on colorectal cancer proliferation, migration and invasion was investigated. Additionally, the effects of SIRT4 on the chemosensitivity in colorectal cancer cells and the underlying molecular mechanisms were also explored. The results demonstrated that SIRT4 expression is significantly downregulated in CRC tissues and cell lines. Downregulation of SIRT4 significantly increased tumor proliferation, migration and invasion. Additionally, downregulation of SIRT4 decreased the chemosensitivity of CRC cells by inhibiting cell apoptosis. Thus, these results suggest that SIRT4 may be a promising therapeutic target in CRC.
\end{abstract}

\section{Introduction}

Colorectal cancer (CRC) is the third most common cause of cancer-associated mortality (1). Despite advances in the diagnosis and therapy, CRC remains a major global health problem (2). Thus, it is necessary to explore the molecular mechanisms underlying the progression of CRC.

Sirtuins (SIRTs) 1-7 are a class of nicotine adenine dinucleotide (NAD)+-dependent deacetylases (3), involved in cellular processes (4), including metabolism (5), stress response $(6,7)$, genomic stability $(8,9)$ and longevity $(10)$. Among SIRTs, SIRT1, SIRT6 and SIRT7 are localized in the nucleus (11-13), SIRT2 resides within the cytoplasm (11), whereas SIRT3, SIRT4 and SIRT5 are mitochondrial $(14,15)$.

Correspondence to: Professor Yanqiao Zhang, Department of Gastrointestinal Medical Oncology, Harbin Medical University Cancer Hospital, 150 Haping Road, Harbin, Heilongjiang 150081, P.R. China E-mail: yanqiaozhang@ems.hrbmu.edu.cn

Key words: sirtuin 4, colorectal cancer, chemosensitivity, apoptosis
Aberrant expression of SIRTs is associated with the pathogenesis of several human diseases, including cancer (16). SIRT4 does not have a NAD+-dependent deacetylase activity but downregulates glutamate dehydrogenase activity (17), an enzyme that converts glutamate to $\alpha$-ketoglutarate and regulates the metabolism of glutamine and glutamate to ultimately produce ATP (18). SIRT4 regulates the balance between fatty acid oxidation and lipid synthesis in skeletal muscle cells and adipocytes $(19,20)$, and regulates insulin secretion in human pancreatic $\beta$ cells $(17,21)$. Additionally, SIRT4 serves a role in the metabolism of amino acids (22). In tumor cells, SIRT4 may negatively regulate cell proliferation by inhibiting the uptake of glutamine (23). Jeong et al (24) reported that SIRT4 regulates the cellular metabolism in response to DNA damage by inhibiting mitochondrial glutamine metabolism. Additionally, decreased protein levels of SIRT4 have been detected in endometrial adenocarcinoma tissues and are associated with advanced American Joint Committee on Cancer (AJCC) stages (25). Downregulation of SIRT4 is associated with poor prognosis in esophageal squamous cell carcinoma (26). Numerous studies demonstrated that SIRT4 is downregulated in several types of human cancer, including breast (27), gastric (28), liver (29), colon (30) and bladder cancer (31) and leukemia (32), however, primarily in lung cancer $(33,34)$, Although previous studies suggested that SIRT4 may act as a tumor suppressor (35), the function of SIRT4 in human cancer remains unclear.

In the present study, the expression of SIRT4 was detected in CRC tissues. Additionally, the effect of SIRT4 downregulation on colon cancer proliferation, migration and invasion was investigated. The effects of SIRT4 on the chemotherapeutic sensitivity of CRC cells and the underlying molecular mechanisms were also explored. The present study provides insights on the role of SIRT4 in CRC progression.

\section{Materials and methods}

Cell lines and cell culture. All cell lines were provided by the Shanghai Institute of Cell Biology (Shanghai, China). The human CRC cell lines HCT116, SW1116, SW620 and DLD1 were maintained in RPMI-1640 (Biological Industries, Kibbutz Beit Haemek, Israel) and the normal colorectal cell line FHC were maintained in Dulbecco's modified Eagle's medium (DMEM; HyClone; GE Healthcare Life Sciences, 
Logan, UT, USA) supplemented with $10 \%$ fetal bovine serum (FBS; Invitrogen; Thermo Fisher Scientific, Inc., Waltham, MA, USA), $100 \mathrm{U} / \mathrm{ml}$ penicillin and $100 \mu \mathrm{g} / \mathrm{ml}$ streptomycin. Cells were cultured at $37^{\circ} \mathrm{C}$ in a humidified atmosphere containing $5 \% \mathrm{CO}_{2}$.

Tissue samples. A total of 30 tissues (15 CRC and 15 matched adjacent normal tissues of male patients) were obtained from the Department of Gastrointestinal Surgical Oncology at Harbin Medical University Cancer Hospital (Heilongjiang, China) between 2011 and 2012. The range years was 38-76 years and the mean age of patients was 52 years. None of the patients received chemotherapy before surgery. All patients signed written informed consent, and the study was approved by the Ethics Committee of Harbin Medical University (Heilongjiang, China).

The Cancer Genome Atlas (TCGA) database. The SIRT4 expression data of 174 samples were downloaded from TCGA data portal on August 2016 (https://cancergenome.nih.gov/). According to the TCGA barcode, the expression data was divided into CRC data and normal data, including 155 CRC tissues and 19 normal colon tissues. The statistical analyses and figures were performed by GraphPad Prism software 5.0 (GraphPad Software, Inc., La Jolla, CA, USA) based on two sets of expression data.

Immunohistochemistry and evaluation of staining. Tissue sections (3.5 $\mu \mathrm{m}$ thick) were prepared from paraffin-embedded tissues. Briefly, sections were deparaffinized in xylene for 5 min (four times) and rehydrated in a descending ethanol series at room temperature. Antigen retrieval was performed using a pressure cooker for $3 \mathrm{~min}$ at $121^{\circ} \mathrm{C}$ in Tris-EDTA buffer. Endogenous peroxidase activity was then blocked by incubation in $3 \%$ hydrogen peroxide for $10 \mathrm{~min}$ at room temperature. The sections were incubated with primary antibody against SIRT4 (ab10140; 1:100; Abcam, Cambridge, UK) overnight at $4^{\circ} \mathrm{C}$. Following the primary incubation, sections were incubated with secondary antibody solution (ZB-2306; 1:5,000; ZSGB-BIO; OriGene Technologies, Inc., Beijing, China) for $1 \mathrm{~h}$ at room temperature. The sections were stained with 3,3-diaminobenzidine tetrahydrochloride (DAB; ZSGB-BIO; OriGene Technologies, Inc.) and counterstained with hematoxylin. Tissues stained with PBS instead of primary antibody, served as negative controls.

The histological evaluation was performed by two pathologists from Harbin Medical University Cancer Hospital (Harbin, China) using a light microscope. The staining score was defined by using intensity $(0$, negative; 1 , weak; 2 , moderate; 3 , strong) and area $(0,<5 \% ; 1,5-25 \% ; 2,25-50 \% ; 3,50-75 \% ; 4,>75 \%)$. The final score was generated by assessing the percentage of area stained multiplied by the intensity of staining.

Reverse transcription-quantitative polymerase chain reaction (RT-qPCR). Total RNA was isolated from tissues and cells with TRIzol reagent (Invitrogen; Thermo Fisher Scientific, Inc.), according to the manufacturer's protocol. RNA was reverse-transcribed into cDNA using TransScript Reverse Transcriptase (AT101-02; Beijing Transgen Biotech Co., Ltd., Beijing, China). qPCR was performed using the SYBR Green
PCR Mix (AQ141-01; Beijing Transgen Biotech, Beijing, China) and the Bio-Rad CFX96TM Real-Time PCR system (Bio-Rad Laboratories, Inc., Hercules, CA, USA). The primers used were as follows: SIRT4, 5'-ATGAAGATGAGCTTT GCGT-3' (forward) and 5'-TCAGCATGGGTCTATCAA AGG-3' (reverse); GAPDH, 5'-ATGGGGAAGGTGAAGGTC G-3' (forward) and 5'-GGGGTCATTGATGGCAACAATA-3' (reverse). GAPDH was used as an endogenous control. The thermocycling conditions were as follows: $95^{\circ} \mathrm{C}$ for $10 \mathrm{~min}$, followed by 40 cycles at $95^{\circ} \mathrm{C}$ for $10 \mathrm{sec}$ and $61^{\circ} \mathrm{C}$ for $30 \mathrm{sec}$. The analysis of RT-qPCR results according to the $2^{-\Delta \Delta \mathrm{Cq}}$ method (36).

Western blot analysis. Total protein was extracted from cells and tissues using a radioimmunoprecipitation assay buffer (Beyotime Institute of Biotechnology, Haimen, China) supplemented with $1 \mathrm{mM}$ phenylmethanesulfonyl fluoride (PMSF, Beyotime Institute of Biotechnology, Shanghai, China). Protein determination was performed using the BCA Protein assay kit (Takara Bio, Inc., Otsu, Japan) and proteins (25 $\mu \mathrm{g})$ were separated by SDS-PAGE (10\% gels) and then transferred onto polyvinylidene difluoride (PVDF) membranes (EMD Millipore, Billerica, MA, USA) and blocked in 5\% bovine serum albumin (Beyotime Institute of Biotechnology) was diluted in PBS for $2 \mathrm{~h}$ at room temperature. Membranes were incubated with anti-SIRT4 (catalog no. ab10140; 1:300, Abcam) or mouse anti- $\beta$-actin (catalog no. sc-58673, 1:5,000; Santa Cruz Biotechnology, Inc., Dallas, TX, USA) antibodies at $4^{\circ} \mathrm{C}$ overnight, followed by incubation with Rabbit Anti-goat IgG H\&L (catalog no. ab6697; 1:5,000; Abcam) or goat anti-mouse IgG H\&L (catalog no. ab6708; 1:5,000; Abcam) for $1 \mathrm{~h}$ at room temperature. The protein bands were visualized by enhanced chemiluminescence (ECL; GE Healthcare Bio-Sciences, Pittsburgh, PA, USA) via FluorChem M imaging system (FCM, FM0422; ProteinSimple, San Jose, CA, USA) and analyzed using the Image J software (https://imagej.nih.gov/ij/).

Apoptosis analysis. Cell apoptosis was evaluated by using Annexin V-phycoerythrin (PE)/7-aminoactinomycin D (7-AAD) Apoptosis Detection kit (BD Biosciences, Franklin Lakes, NJ, USA), according to the manufacturer's protocol. Briefly, a total of $1 \times 10^{6}$ HCT116 cells were collected and washed with PBS, and then the cells were resuspended with 1-ml Binding Buffer. $100 \mu 1$ solution $\left(1 \times 10^{5}\right), 5 \mu 1 \mathrm{PE}$ Annexin $\mathrm{V}$ and $5 \mu \mathrm{l}$ 7-ADD were added in a $5 \mathrm{ml}$ tube. The cells were vortexed gently and then incubated for $10 \mathrm{~min}$ in the dark at room temperature. At last, $400 \mu$ l Binding Buffer was added in each tube before analyzed by flow cytometry. The flow cytometry data were analyzed by software (FlowJo 7.6.1; FlowJo LLC, Ashland, OR, USA).

Cell viability assay. Cellular viability was evaluated using CellTiter-Glo assay (Promega Corporation, Madison, WI, USA), according to the manufacturer's protocol. Briefly, HCT116 cells in RPMI-1640 (Biological Industries, Kibbutz Beit Haemek, Israel) were plated in 96-well plates at a density of $1 \times 10^{4}$ cells/well at room temperature. At 0, 24, 48 and $72 \mathrm{~h}$, $100 \mu \mathrm{l}$ CellTiter-Glo reagent was added into each well and mixed completely using an orbital shaker at room temperature. Following incubation for $10 \mathrm{~min}$ at room temperature, the luminescence signal was assessed using a luminometer. 
Clustered Regularly Interspaced Short Palindromic Repeats (CRISPR)/CRISPR-associated protein-9 nuclease (Cas9)-mediated knockout of SIRT4 in HCT116 cells. In the present study a CRISPR-Cas9 vector, targeting specific region of SIRT4 (5'-CCGAATCGGGGATACCAGAC-3') was used. Guide RNA sequence for CRISPR/Cas9 was designed using the CRISPR Design Tool (http://tools.genome-engineering. org) on August 2016. HCT116 cells were grown to 80-90\% confluence and transfected with CRISPR plasmids (pSpCas9) by using Lipofectamine 2000 (Invitrogen; Thermo Fisher Scientific, Inc.) at room temperature. Cells were treated with $2 \mu \mathrm{g} / \mathrm{ml}$ puromycin (ST551, Beyotime Institute of Biotechnology, Shanghai, China) for $24 \mathrm{~h}$. Transfected cells were sorted to obtain single cell clones. Western blot analysis was used to confirm the knockout of SIRT4 in HCT116 cells.

Invasion and migration assay. Cell migration was evaluated using a Transwell chamber without (3422, Corning, USA) according to the manufacturer's protocol. Cell invasion was evaluated using a Transwell chamber with Matrigel (Corning Incorporated, Corning, NY, USA) according to the manufacturer's protocol. For the upper chambers, $3 \times 10^{5}$ cells $/ \mathrm{ml}$ were suspended in $0.5 \mathrm{ml}$ of serum-free DMEM. The lower chamber contained DMEM supplemented with 10\% FBS. Plates were incubated at $37^{\circ} \mathrm{C}$ in a humidified atmosphere containing $5 \% \mathrm{CO}_{2}$. At $48 \mathrm{~h}$, cells remaining on the upper membrane surface were removed and the invasive cells on the lower surface were fixed with $1 \%$ crystal violet for $30 \mathrm{~min}$ and counted at room temperature. Images were captured using an Olympus inverted microscope (Olympus Corporation, Tokyo, Japan). The number of invaded cells was counted using ImageJ software (National Institutes of Health, MD, Bethesda, USA).

5-FU treatment. 5-fluorouracil $0.25 \mathrm{~g} / 10 \mathrm{ml}$ or oxaliplatin $50 \mathrm{mg}$ was purchased from Harbin Medical University Cancer Hospital and storage at $4^{\circ} \mathrm{C}$. HCT116 cells were treated with 5-FU for $36 \mathrm{~h}$ at $37^{\circ} \mathrm{C}$ and treated HCT116 cells with oxaliplatin for $48 \mathrm{~h}$ at $37^{\circ} \mathrm{C}$.

Statistical analysis. Data were analyzed using GraphPad Prism (version 5.0; GraphPad Software, Inc.). Data are expressed as the mean \pm standard deviation. Three individual experiments were performed. Results were analyzed using Student's t-test or one-way ANOVA analysis, following the analysis of variance. $\mathrm{P}<0.05$ was considered to indicate a statistically significant difference.

\section{Results}

Expression of SIRT4 in CRC based on data obtained from TCGA.The SIRT4 expression data from 174 samples (including 19 normal colon tissues and 155 CRC tissues) were downloaded from TCGA data portal. The results demonstrated that the expression of SIRT4 was significantly decreased in colon adenocarcinoma tissues compared with normal colon tissues (Fig. 1).

SIRT4 is downregulated in CRC cell lines and tissues. mRNA and protein expression levels of SIRT4 were examined in CRC cell lines and tissues. The results confirmed that mRNA expression

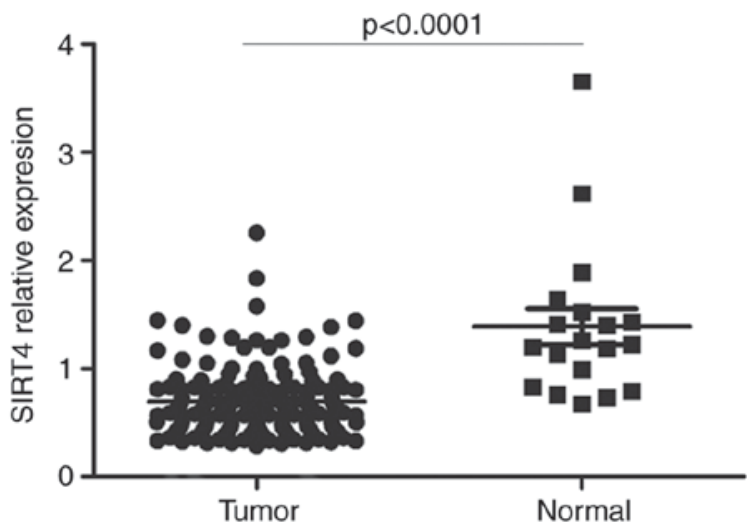

Figure 1. The expression of SIRT4 was evaluated in CRC and normal tissues using data obtained from TCGA. SIRT, sirtuin; CRC, colorectal cancer; TCGA, The Cancer Genome Atlas.

levels of SIRT4 were significantly decreased compared with that in adjacent normal tissues (Fig. 2A). Additionally, mRNA expression levels of SIRT4 were decreased in CRC cell lines (HCT116, SW1116, SW620 and DLD1) compared with that in the normal colorectal cell line FHC (Fig. 2B). Protein expression of SIRT4 was evaluated in tissues using western blot analysis and immunohistochemistry. The results demonstrated that the expression level of SIRT4 in CRC tissues was decreased compared with that in adjacent normal tissues (Fig. 2C-E). Thus, SIRT4 is downregulated in CRC tissues and cells, suggesting that SIRT4 may be involved in the pathogenesis of CRC.

SIRT4 functions as a potential tumor suppressor by repressing the proliferation, and migration of CRC cells. To determine the function of SIRT4 in colorectal cancer, CRISPR/Cas9-mediated knockout of SIRT4 was performed in HCT116 cells. The knockout of SIRT4 was confirmed using western blot analysis (Fig. 3A). The proliferative ability of control cells $\left(\right.$ HCT116 SIRT4 ${ }^{+/+}$) and SIRT4 knockout cells $\left(\right.$ HCT116 SIRT4 ${ }^{-/}$) was evaluated. The results demonstrated that HCT116 SIRT4 ${ }^{--}$cells grew faster compared with HCT116 SIRT4 $^{+/+}$cells (Fig. 3B). Additionally, the effect of SIRT4 knockout on the migratory abilities was determined in HCT116 cells. The results demonstrated that HCT116 SIRT4 ${ }^{-/}$significantly enhanced the migratory ability (Fig. 3C) and invasive ability (Fig. 3D). Therefore, the knockout of SIRT4 promoted the proliferation, migration and invasion of HCT116 cells.

Knockout of SIRT4 decreases chemosensitivity in CRC cells by inhibiting apoptosis. The effects of SIRT4 knockout in regulating chemotherapy in CRC were also evaluated. HCT116 SIRT4 $^{-/}$and HCT116 SIRT4 $^{+/+}$were treated with various concentrations of 5-fluorouracil (5-FU) or oxaliplatin for $48 \mathrm{~h}$ at $37^{\circ} \mathrm{C}$. CellTiter-Glo Luminescent assay was employed to determine the effect of SIRT4 on the chemosensitivity of CRC cells. The results demonstrated that the viability of HCT116 $\mathrm{SIRT}^{-/-}$cells was increased compared with that in SIRT4 ${ }^{+/+}$ in response to treatment with 5-FU or oxaliplatin by inhibition percent (\%) (Fig. 4A and B). These results suggest that knockout of SIRT4 may decrease the chemosensitivity of CRC cells. Next, the effect of SIRT4 knockout on cells apoptosis was evaluated. Due to the high drug concentration required 

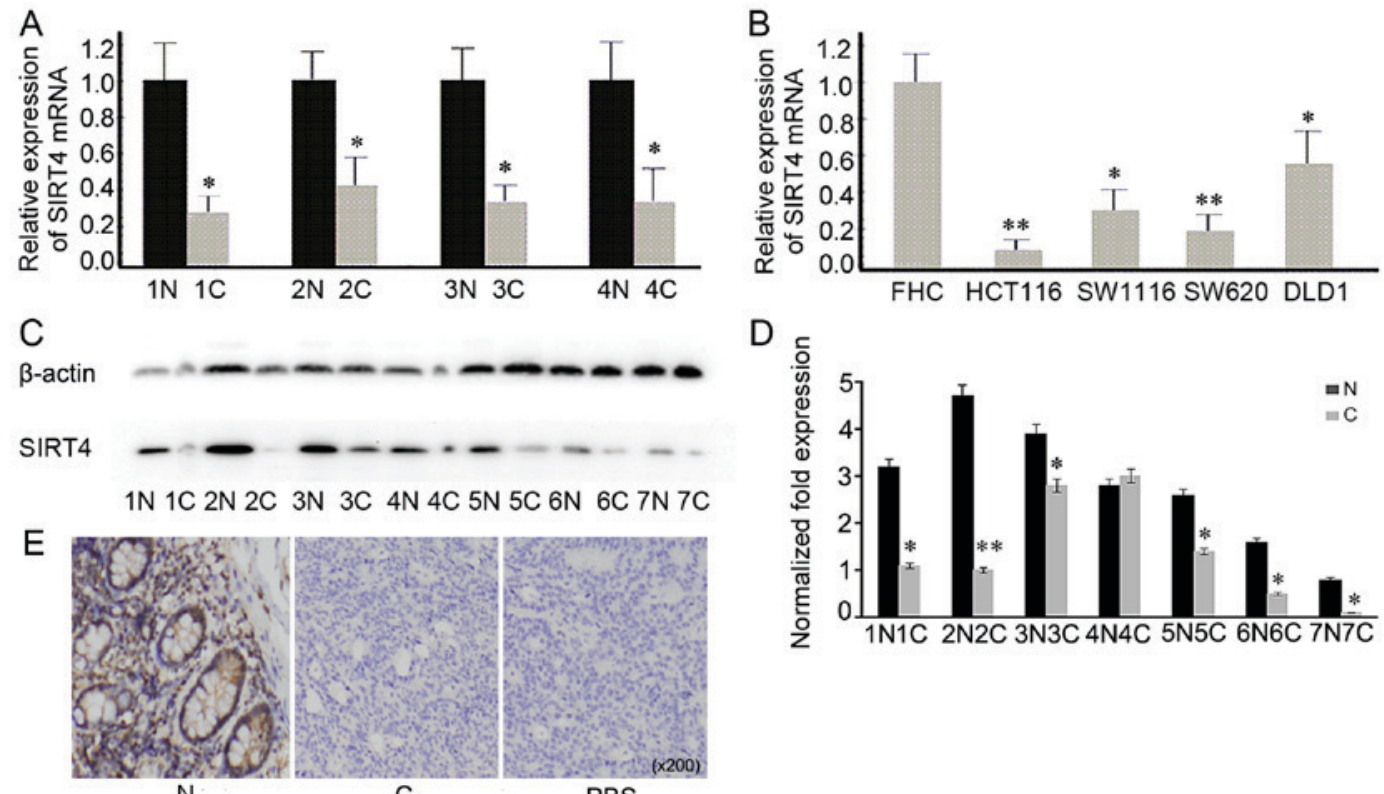

1N1C 2N2C 3N3C 4N4C 5N5C 6N6C 7N7C

Figure 2. Detection of SIRT4 expression in CRC tissues and cell lines. (A) mRNA expression levels of SIRT4 in representative CRC tissues and adjacent normal tissues was examined. "P $<0.05$ vs. N. (B) mRNA expression levels of SIRT4 in CRC cell lines (HCT116, SW1116, SW620 and DLD1) was decreased compared with that in the normal cell line FHC. ${ }^{* *} \mathrm{P}<0.01,{ }^{*} \mathrm{P}<0.05$ vs. FHC. (C) The expression of SIRT4 in seven CRC tissues and adjacent normal tissues was evaluated using western blot analysis. (D) The densitometric analysis of SIRT4 expression protein was downregulated in CRC tissues compared with that in normal tissues. ${ }^{* *} \mathrm{P}<0.01,{ }^{*} \mathrm{P}<0.05$ vs. N. (E) Representative immunohistochemical staining of SIRT4 in human CRC and adjacent normal tissues. SIRT4 was mainly expressed in the cytoplasm, and its expression was decreased in CRC tissues compared with that in adjacent normal tissue. SIRT, sirtuin; CRC, colorectal cancer; N, normal tissue; C, CRC tissue; PBS, negative control.

\section{A}
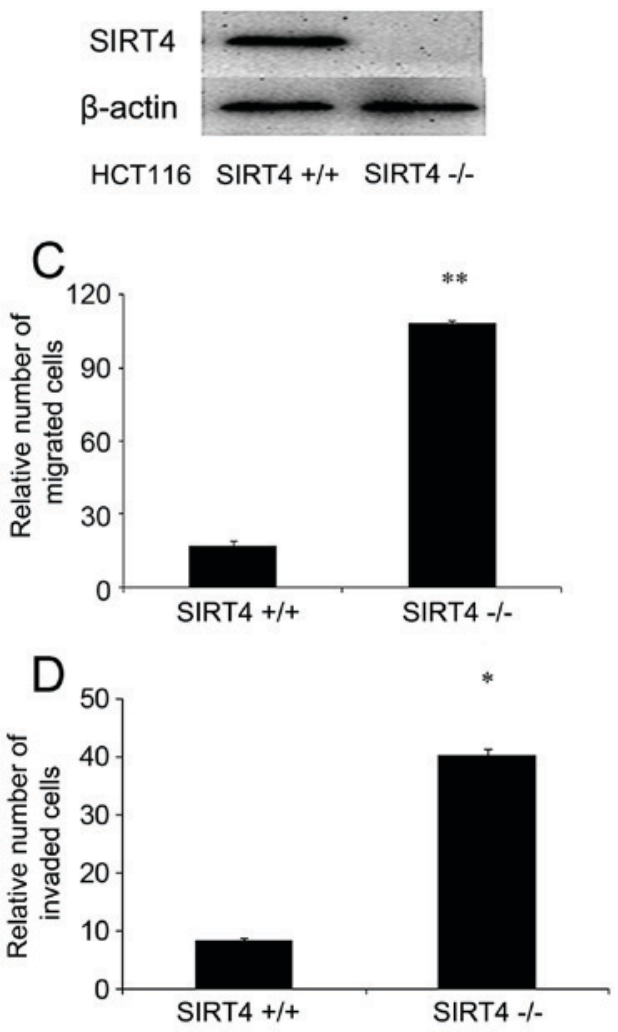
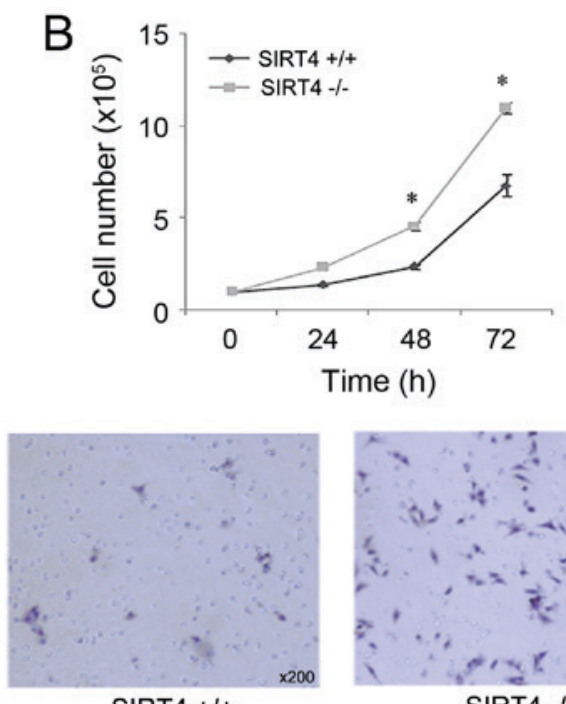

SIRT4 +1+

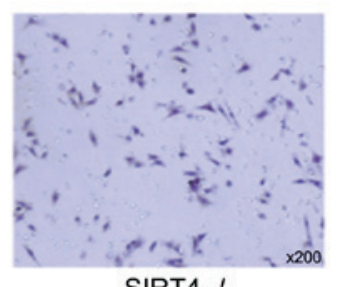

SIRT4 -/-

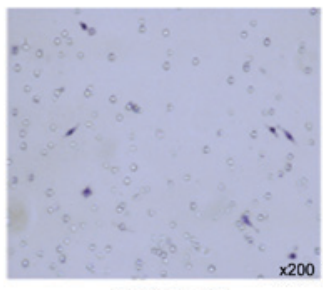

SIRT4 +/+

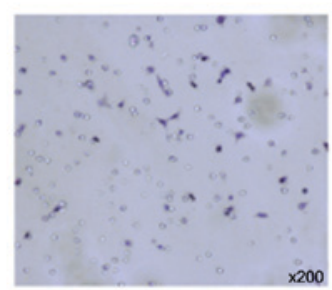

SIRT4 -/-

Figure 3. SIRT4 regulates proliferation and migration in CRC cells. (A) Knockout of SIRT4 in HCT116 cells was confirmed by western blot analysis. (B) HCT116 SIRT4 $\%$ cells exhibited a significant increase in proliferation. " $\mathrm{P}<0.05 \mathrm{vs}$. HCT116 SIRT4 $\%$. (C) Knockout of SIRT4 promoted migration of HCT116 cells by counting numbers of migrating cells (magnification, $\mathrm{x} 200$ ). ${ }^{* *} \mathrm{P}<0.01 \mathrm{vs}$. HCT116 SIRT4 ${ }^{-1}$. (D) Knockout of SIRT4 promoted invasion of HCT116 cell by counting numbers of invasive cells (magnification, x200). "P<0.05 vs. HCT116 SIRT4 ${ }^{-1}$. SIRT, sirtuin; CRC, colorectal cancer; HCT116 SIRT4 ${ }^{+/+}$, wild-type; HCT116 SIRT4 ${ }^{--}$, SIRT4 knockout. 

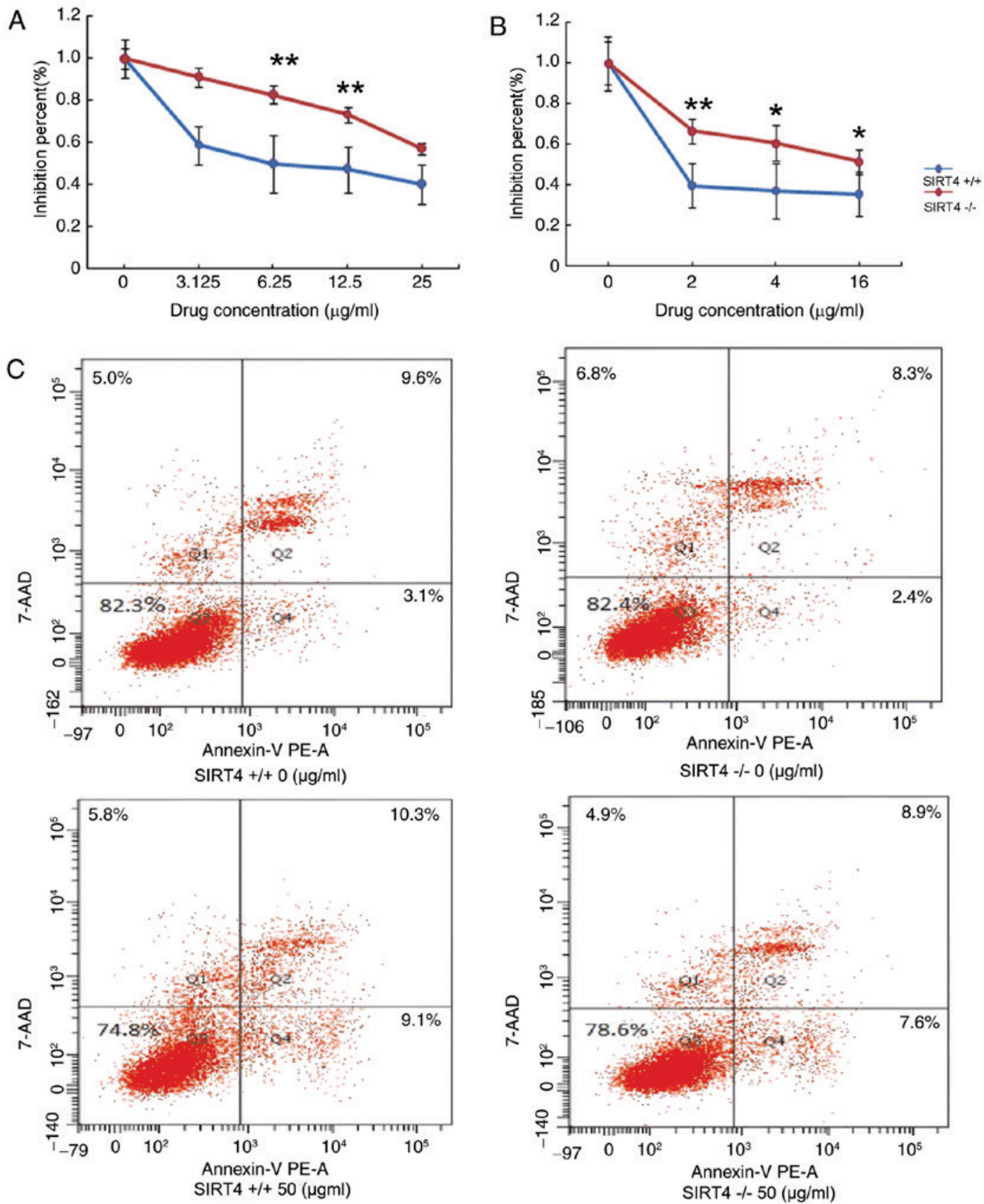

Figure 4. Knockout of SIRT4 decreases the chemotherapeutic sensitivity of CRC cells to 5-FU or oxaliplatin by inhibiting apoptosis. (A) The viability of HCT116 SIRT4 ${ }^{-/}$cells is decreased compared with HCT116 SIRT4 ${ }^{+/+}$in response to 5-FU treatment by evaluating inhibition percent $(\%){ }^{* *}$ P $<0.01$ vs. HCT116 SIRT4 $^{-/}$. (B) The viability of HCT116 SIRT4 ${ }^{-/}$cells is decreased compared with HCT116 SIRT4 ${ }^{+/}$in response to oxaliplatin treatment by evaluating inhibition percent $(\%),{ }^{* *} \mathrm{P}<0.01$ vs. HCT116 SIRT4 ${ }^{-/},{ }^{*} \mathrm{P}<0.05$ vs. HCT116 SIRT4 ${ }^{-/}$. (C) SIRT4 knockout inhibits apoptosis in response to 5-FU. SIRT, sirtuin; HCT116 SIRT4 $^{+/+}$, wild-type; HCT116 SIRT4 ${ }^{-/}$, SIRT4 knockout; 7-AAD, 7-aminoactinomycin D; PE, phycoerythrin; 5-FU, 5-fluorouracil.

for apoptosis, HCT116 SIRT4 ${ }^{-/-}$cells and HCT116 SIRT4 $^{+/+}$ cells were treated with $50 \mu \mathrm{g} / \mathrm{ml} 5$-FU and cell apoptosis was evaluated using flow cytometry. The results demonstrated that the apoptosis rate was decreased in HCT116 SIRT4 $^{-/}$cells compared with that of HCT116 SIRT4 $^{+/+}$cells (Fig. 4C), suggesting that knockout of SIRT4 may decrease sensitivity to chemotherapy by inhibiting apoptosis in CRC cells.

\section{Discussion}

Previous studies demonstrated that SIRT4 is downregulated in various cancer types $(23,24)$ and is associated with multiple cancer biological behaviors (23). However, the function of SIRT4 in CRC remains unclear. The results of the present study revealed that the expression of SIRT4 was significantly decreased in CRC tissues and cell lines at mRNA and protein levels, which is consistent with previous studies. Previous studies revealed that SIRT4 might function as a tumor suppressor. Jeong et al (24) demonstrated that SIRT4 may suppress tumor formation by inhibiting glutamine metabolism, and overexpression of SIRT4 may prevent the growth of HeLa cells. Additionally, Csibi et al (23) revealed that overexpression of SIRT4 may prevent the grow th of the prostate cancer cell line DU145 and the colon 
cancer cell line DLD-1. Jeong et al (32) revealed that SIRT4 might inhibit the growth of Myc-induced B lymphoma cell by inhibiting glutamine metabolism. In the present study, stable human SIRT4 KO cells were established and the effects of knockout of SIRT4 on proliferation, migration and invasion of CRC cells were examined. The results demonstrated that knockout of SIRT4 increased the proliferation, migration and invasion of CRC cells, thus confirming the findings of previous studies (30).

Targeting SIRT4 may be a novel therapeutic approach in CRC. Chemotherapy is considered as one of the most effective treatment approaches in CRC (37). 5-FU and oxaliplatin are widely used for the treatment of CRC (38). 5-FU-based chemotherapy in combination with oxaliplatin or irinotecan is used for the treatment of metastatic CRC (39). 5-FU metabolites are incorporated into DNA to suppress cell growth (40). In tumor cells, 5-FU is metabolized to cytotoxic compounds, which bind to thymidylate synthase in order to repress DNA synthesis (41). Oxaliplatin, a platinum-based chemotherapeutic drug, containing 1,2-diaminocyclohexane carrier ligand, forms platinum-DNA adducts, which block DNA replication. In addition, oxaliplatin has demonstrated high efficacy against tumor growth both in vitro and in vivo (42). Oxaliplatin treatment combined with 5-FU increases progression-free survival and overall survival of CRC (43). Oxaliplatin and 5-FU may act synergistically to regulate thymidylate synthase (44). In the present study, the effects SIRT4 on the chemosensitivity of CRC cells were evaluated. To the best of our knowledge, this is the first study to examine the role of SIRT4 in cancer chemotherapy. The results demonstrated that SIRT4 KO cells exhibited increased viability compared with control cells, suggesting that SIRT4 may enhance the chemosensitivity of CRC cells to 5-FU and oxaliplatin. Next, the underlying molecular mechanism was investigated. A number of events involved in apoptosis are closely associated with mitochondria, including the release of caspase activators, loss of mitochondrial transmembrane potential, altering electron transport, and participation of Bcl-2 family proteins $(45,46)$. SIRT4 is located in the mitochondria. Previous studies have demonstrated that SIRT4 serves an important function in hypoxia-induced apoptosis in H9c2 cardiomyoblast cells through affecting Bcl-2-associated-X protein (Bax) translocation (47). Therefore, it was hypothesized that SIRT4 may affect the chemotherapeutic sensitivity of CRC cells through regulating apoptosis. The results revealed that SIRT4 knockout prevents the apoptosis of CRC cells in response to 5-FU. These results suggest that SIRT4 may act as a tumor suppressor and may be used as a novel therapeutic target in CRC.

In conclusion, the present study demonstrated that the expression of SIRT4 is significantly decreased in CRC tissues at mRNA and protein levels, and that SIRT4 knockout increases tumor proliferation, migration and invasion, thus suggesting that SIRT4 may act as a tumor suppressor. Additionally, SIRT4 knockout affects the chemotherapeutic sensitivity of CRC cells by regulating apoptosis. The present study provides a promising target for CRC therapy.

\section{Acknowledgements}

Not applicable.

\section{Funding}

The present study was supported by the National Natural Science Foundation of China (grant no. 81172265).

\section{Availability of data and materials}

The data used and analyzed during the current study are available from the corresponding author on reasonable request.

\section{Authors' contributions}

YaZ conceived and designed the experiments. YuZ, GW, XL, TW and MW performed the experiments and analyzed the data. YuZ and YaZ wrote the paper. All authors read and approved the final manuscript.

\section{Ethics approval and consent to participate}

All patients provided written informed consent prior to their inclusion and the present study was approved by the Ethics Committee of Harbin Medical University (Heilongjiang, China).

\section{Consent for publication}

This research was completed in compliance with the Helsinki Declaration. The patients provided written informed consent for the publication of the data. The data collection and analysis were carried out without disclosing the identities of the patients.

\section{Competing interests}

The authors declare that they have no competing interests.

\section{References}

1. Siegel RL, Miller KD and Jemal A: Cancer statistics, 2015. CA Cancer J Clin 65: 5-29, 2015.

2. Siegel RL, Miller KD, Fedewa SA, Ahnen DJ, Meester RGS, Barzi A and Jemal A: Colorectal cancer statistics, 2017. CA Cancer J Clin 67: 177-193, 2017.

3. Argmann C and Auwerx J: Insulin secretion: SIRT4 gets in on the act. Cell 126: 837-839, 2006.

4. Finkel T, Deng CX and Mostoslavsky R: Recent progress in the biology and physiology of sirtuins. Nature 460: 587-591, 2009.

5. Kleszcz R, Paluszczak J and Baer-Dubowska W: Targeting aberrant cancer metabolism-The role of sirtuins. Pharmacol Rep 67 1068-1080, 2015.

6. Jeong SM, Hwang S and Seong RH: SIRT4 regulates cancer cell survival and growth after stress. Biochem Biophys Res Commun 470: 251-256, 2016.

7. Feng J, Yan PF, Zhao HY, Zhang FC, Zhao WH and Feng M: SIRT6 suppresses glioma cell growth via induction of apoptosis, inhibition of oxidative stress and suppression of JAK2/STAT3 signaling pathway activation. Oncol Rep 35: 1395-1402, 2016.

8. Kiran S, Oddi V and Ramakrishna G: Sirtuin 7 promotes cellular survival following genomic stress by attenuation of DNA damage, SAPK activation and p53 response. Exp Cell Res 331: 123-141, 2015.

9. Kim HS, Vassilopoulos A, Wang RH, Lahusen T, Xiao Z, Xu X, Li C, Veenstra TD, Li B, Yu H, et al: SIRT2 maintains genome integrity and suppresses tumorigenesis through regulating APC/C activity. Cancer Cell 20: 487-499, 2011.

10. Benigni A, Perico L and Macconi D: Mitochondrial dynamics is linked to longevity and protects from End-organ injury: The emerging role of Sirtuin 3. Antioxid Redox Signal 25: 185-199, 2016. 
11. Singh S, Kumar PU, Thakur S, Kiran S, Sen B, Sharma S, Rao VV, Poongothai AR and Ramakrishna G: Expression/localization patterns of sirtuins (SIRT1, SIRT2, and SIRT7) during progression of cervical cancer and effects of sirtuin inhibitors on growth of cervical cancer cells. Tumour Biol 36: 6159-6171, 2015 .

12. Azuma Y, Yokobori T, Mogi A, Altan B, Yajima T, Kosaka T, Onozato R, Yamaki E, Asao T, Nishiyama M and Kuwano H: SIRT6 expression is associated with poor prognosis and chemosensitivity in patients with non-small cell lung cancer. J Surg Oncol 112: 231-237, 2015

13. Zhang PY, Li G, Deng ZJ, Liu LY, Chen L, Tang JZ, Wang YQ, Cao ST, Fang YX, Wen F, et al: Dicer interacts with SIRT7 and regulates $\mathrm{H} 3 \mathrm{~K} 18$ deacetylation in response to DNA damaging agents. Nucleic Acids Res 44: 3629-3642, 2016.

14. Padmaja Divya S, Pratheeshkumar P, Son YO, Vinod Roy R, Andrew Hitron J, Kim D, Dai J, Wang L, Asha P, Huang B, et al: Arsenic induces insulin resistance in mouse adipocytes and myotubes via oxidative stress-regulated mitochondrial Sirt3-FOXO3a signaling pathway. Toxicol Sci 146: 290-300, 2015.

15. Pacella-Ince L, Zander-Fox DL and Lane M: Mitochondrial SIRT5 is present in follicular cells and is altered by reduced ovarian reserve and advanced maternal age. Reprod Fertil Dev 26: 1072-1083, 2014

16. Yuan H, Su L and Chen WY: The emerging and diverse roles of sirtuins in cancer: A clinical perspective. Onco Targets Ther 6: 1399-1416, 2013

17. Ahuja N, Schwer B, Carobbio S, Waltregny D, North BJ, Castronovo V, Maechler P and Verdin E: Regulation of insulin secretion by SIRT4, a mitochondrial ADP-ribosyltransferase. J Biol Chem 282: 33583-33592, 2007.

18. Ho L, Titus AS, Banerjee KK, George S, Lin W, Deota S, Saha AK, Nakamura K, Gut P, Verdin E and Kolthur-Seetharam U: SIRT4 regulates ATP homeostasis and mediates a retrograde signaling via AMPK. Aging (Albany NY) 5: 835-849, 2013

19. Laurent G, German NJ, Saha AK, de BoerVC,Davies M,Koves TR, Dephoure N, Fischer F, Boanca G, Vaitheesvaran B, et al: SIRT4 coordinates the balance between lipid synthesis and catabolism by repressing malonyl CoA decarboxylase. Mol Cell 50: 686-698, 2013.

20. Nasrin N, Wu X, Fortier E, Feng Y, Bare' OC, Chen S, Ren X, Wu Z, Streeper RS and Bordone L: SIRT4 regulates fatty acid oxidation and mitochondrial gene expression in liver and muscle cells. J Biol Chem 285: 31995-32002, 2010.

21. Haigis MC, Mostoslavsky R, Haigis KM, Fahie K, Christodoulou DC, Murphy AJ, Valenzuela DM, Yancopoulos GD, Karow M, Blander G, et al: SIRT4 inhibits glutamate dehydrogenase and opposes the effects of calorie restriction in pancreatic beta cells. Cell 126: 941-954, 2006

22. Houtkooper RH, Pirinen E and Auwerx J: Sirtuins as regulators of metabolism and healthspan. Nat Rev Mol Cell Biol 13. 225-238, 2012

23. Csibi A, Fendt SM, Li C, Poulogiannis G, Choo AY, Chapski DJ, Jeong SM, Dempsey JM, Parkhitko A, Morrison T, et al: The mTORC1 pathway stimulates glutamine metabolism and cell proliferation by repressing SIRT4. Cell 153: 840-854, 2013.

24. Jeong SM, Xiao C, Finley LW, Lahusen T, Souza AL, Pierce K, Li YH, Wang X, Laurent G, German NJ, et al: SIRT4 has tumor-suppressive activity and regulates the cellular metabolic response to DNA damage by inhibiting mitochondrial glutamine metabolism. Cancer Cell 23: 450-463, 2013.

25. Chen X, Lai X, Wu C, Tian Q, Lei T, Pan J and Huang G: Decreased SIRT4 protein levels in endometrioid adenocarcinoma tissues are associated with advanced AJCC stage. Cancer Biomark 19: 419-424, 2017

26. Nakahara Y, Yamasaki M, Sawada G, Miyazaki Y, Makino T, Takahashi T, Kurokawa Y, Nakajima K, Takiguchi S, Mimori K, et al: Downregulation of SIRT4 expression is associated with poor prognosis in esophageal squamous cell carcinoma. Oncology 90: 347-355, 2016

27. Igci M, Kalender ME, Borazan E, Bozgeyik I, Bayraktar R, Bozgeyik E, Camci C and Arslan A: High-throughput screening of Sirtuin family of genes in breast cancer. Gene 586: 123-128, 2016.

28. Huang G, Cui F, Yu F, Lu H, Zhang M, Tang H and Peng Z: Sirtuin-4 (SIRT4) is downregulated and associated with some clinicopathological features in gastric adenocarcinoma. Biomed Pharmacother 72: 135-139, 2015.
29. Wang JX, Yi Y, Li YW, Cai XY, He HW, Ni XC, Zhou J, Cheng YF, Jin JJ, Fan J and Qiu SJ: Down-regulation of sirtuin 3 is associated with poor prognosis in hepatocellular carcinoma after resection. BMC Cancer 14: 297, 2014.

30. Miyo M, Yamamoto H, Konno M, Colvin H, Nishida $N$, Koseki J, Kawamoto K, Ogawa H, Hamabe A, Uemura M, et al: Tumour-suppressive function of SIRT4 in human colorectal cancer. Br J Cancer 113: 492-499, 2015.

31. Blaveri E, Simko JP, Korkola JE, Brewer JL, Baehner F, Mehta K, Devries S, Koppie T, Pejavar S, Carroll P and Waldman FM: Bladder cancer outcome and subtype classification by gene expression. Clin Cancer Res 11: 4044-4055, 2005.

32. Jeong SM, Lee A, Lee J and Haigis MC: SIRT4 protein suppresses tumor formation in genetic models of Myc-induced B cell lymphoma. J Biol Chem 289: 4135-4144, 2014

33. Fu L, Dong Q, He J, Wang X, Xing J, Wang E, Qiu X and Li Q SIRT4 inhibits malignancy progression of NSCLCs, through mitochondrial dynamics mediated by the ERK-Drpl pathway. Oncogene 36: 2724-2736, 2017

34. Garber ME, Troyanskaya OG, Schluens K, Petersen S, Thaesler Z, Pacyna-Gengelbach M, van de Rijn M, Rosen GD, Perou CM, Whyte RI, et al: Diversity of gene expression in adenocarcinoma of the lung. Proc Natl Acad Sci USA 98: 13784-13789, 2001.

35. Zhu Y, Yan Y, Principe DR, Zou X, Vassilopoulos A and Gius D: SIRT3 and SIRT4 are mitochondrial tumor suppressor proteins that connect mitochondrial metabolism and carcinogenesis. Cancer Metab 2: 15, 2014

36. Schmittgen TD and Livak KJ: Analyzing real-time PCR data by the comparative C(T) method. Nat Protoc 3: 1101-1108, 2008

37. Lombardi L, Morelli F, Cinieri S, Santini D, Silvestris N, Fazio N, Orlando L, Tonini G, Colucci G and Maiello E: Adjuvant colon cancer chemotherapy: Where we are and where we'll go. Cancer Treat Rev 36 (Suppl 3): S34-S41, 2010.

38. AlShamaileh H, Wang T, Xiang D, Yin W, Tran PH, Barrero RA, Zhang PZ, Li Y, Kong L, Liu K, et al: Aptamer-mediated survivin RNAi enables 5-fluorouracil to eliminate colorectal cancer stem cells. Sci Rep 7: 5898, 2017

39. Siesing C, Sorbye H, Dragomir A, Pfeiffer P, Qvortrup C, Pontén F, Jirström K, Glimelius B and Eberhard J: High RBM3 expression is associated with an improved survival and oxaliplatin response in patients with metastatic colorectal cancer. PLoS One 12: e0182512, 2017.

40. Noordhuis P, Holwerda U, Van Laar JA, Van der Wilt CL and Peters GJ: A non-radioactive sensitive assay to measure 5-fluorouracil incorporation into DNA of solid tumors. Nucleosides Nucleotides Nucleic Acids 23: 1481-1484, 2004.

41. Chen Q, Meng F, Wang L, Mao Y, Zhou H, Hua D, Zhang H and Wang W: A polymorphism in ABCC4 is related to efficacy of 5-FU/capecitabine-based chemotherapy in colorectal cancer patients. Sci Rep 7: 7059, 2017.

42. Raymond E, Faivre S, Woynarowski JM and Chaney SG: Oxaliplatin: Mechanism of action and antineoplastic activity. Semin Onco 25 (2 Suppl 5): S4-S12, 1998.

43. Douillard JY, Cunningham D, Roth AD, Navarro M, James RD, Karasek P, Jandik P, Iveson T, Carmichael J, Alakl M, et al: Irinotecan combined with fluorouracil compared with fluorouracil alone as first-line treatment for metastatic colorectal cancer: A multicentre randomised trial. Lancet 355: 1041-1047, 2000.

44. Raymond E, Faivre S, Chaney S, Woynarowski J and Cvitkovic E: Cellular and molecular pharmacology of oxaliplatin. Mol Cancer Ther 1: 227-235, 2002.

45. Wang L, Zhou H, Wang Y, Cui G and Di LJ: CtBP maintains cancer cell growth and metabolic homeostasis via regulating SIRT4. Cell Death Dis 6: e1620, 2015.

46. Verdin E, Hirschey MD, Finley LW and Haigis MC: Sirtuin regulation of mitochondria: Energy production, apoptosis, and signaling. Trends Biochem Sci 35: 669-675, 2010.

47. Liu B, Che W, Xue J, Zheng C, Tang K, Zhang J, Wen J and Xu Y: SIRT4 prevents hypoxia-induced apoptosis in H9c2 cardiomyoblast cells. Cell Physiol Biochem 32: 655-662, 2013.

This work is licensed under a Creative Commons Attribution-NonCommercial-NoDerivatives 4.0 International (CC BY-NC-ND 4.0) License. 